\title{
Smart Education as Empowerment: Outlining Veteran Teachers' Training to Promote Digital Migration
}

\author{
José Carlos Morgado ${ }^{1}$ (D) . José Alberto Lencastre ${ }^{1}$ (D) Thiago Freires $^{1}$ (D) . \\ Marco Bento $^{1}$ (D)
}

Accepted: 7 January 2021

(c) The Author(s), under exclusive licence to Springer Nature B.V. part of Springer Nature 2021

\begin{abstract}
Within the enhancement of technology and its ongoing integration into formal education setting, learning environments have been challenged to operationalize and arrange systems that engage pedagogy and technology together. The nature of this ongoing transformation is closely related to the paradigms that reign in the twenty-first century, in a scenario of what is now called a Fourth Industrial Revolution. School, despite losing its monopoly on knowledge diffusion, still plays a central role in educating new generations, therefore, it holds key responsibility in addressing contemporary logics of learning, living and becoming a citizen. Amidst the course of change and the ultimate calls for innovation in education, we encounter veteran teachers, professionals with a long teaching history, whose challenges include becoming familiar with new devices in order to fulfil their work demands. In this article, we then explore how central veteran teachers are for the progression to a smarter education scenario, through debating a training carried out in Portugal with 38 teachers from pre-school and k-12, aimed at promoting their digital migration. Data strengthen ties regarding teachers' perceptions and attitudes in relation to technology and consequent resource on it as pedagogical tools. Also, the overall discussion of the training provides clues on how teacher-oriented actions might address their identity if meaningful output is desired, in order to support a real change of practice.
\end{abstract}

Keywords Smart education · Digital migration · Veteran teachers · Teacher training • Formal education

Thiago Freires

freiresle@gmail.com

José Carlos Morgado

jmorgado@ie.uminho.pt

José Alberto Lencastre

jlencastre@ie.uminho.pt

Marco Bento

macbento@hotmail.com

1 Institute of Education, University of Minho, Braga, Portugal 


\section{Introduction}

Of the many challenges we face nowadays, one of the most intense relates to the daily use of technology. We are at the heart of a technology revolution that is changing the way we live and relate to one another. Schwab (2016) conceptualizes this moment as the Fourth Industrial Revolution, highlighting its provision of a social complexity that humankind has not experienced before. A reflection of such complexity is contained in the unlimited possibilities of connecting billions of people via mobile devices, giving rise to unprecedented access to knowledge, for instance. Admittedly, we still need to understand the speed and magnitude of this new revolution. While the profound uncertainty surrounding the development and adoption of emerging technologies means that we do not yet know how the transformations driven by this revolution will unfold, on the societal front, a paradigm shift is underway, already impacting on how we work and communicate.

In the educational system, institutions have experienced the influence of the arising digital world and despite some scepticism, changes have also taken place, both in pedagogical and curricular dimensions (Baruch and Erstad 2018; Camilleri and Camilleri 2017). New ways to teach and learn with smart technology promise change and rebirth, to some extent. Reaching an overall transformation, though, implies an array of massive demands, ranging from professional development issues to the capability of adapting infrastructure (e.g. Bingimlas, 2009). Among the challenges, a crucial one regards the acknowledgment and training of veteran teachers-professionals with a long teaching history-into the framework of a smart technology world. These teachers make up a considerable portion of the teaching force in OCDE countries, overreaching a forty percent mark in Portugal, New Zealand, Italy and Estonia, for example (OECD 2019a), then, constituting a subject of increased interest.

In this article, we focus on the presentation and discussion of the formative model regarding training of veteran teachers on the grounds of technology-enhanced learning (TEL), with the perspective of fostering their mobility towards a smart education framework. Initially, we make an effort to establish the roots of «smart» as a concept in education, then proceeding to the defence that teachers play a central role in the development of smart learning environments, being strategic decision makers both in outlining and defining curriculum practices. Presenting the context in which this research is carried out, the Rekindle +50 project, we pinpoint our teaching training model, discuss its potentialities as a means for promoting transformation in k-12 educational setting and beyond, bearing in mind teachers' perspectives and participation.

\subsection{Acknowledging the «Smart» in Educational Setting}

Smart education is a concept under formation that implies the resource of education on smart devices along with cutting-edge ICT oriented for teaching and learning (Hoel and Mason 2018; Jo et al. 2014). Making up a currently growing market around the world (Zhu et al. 2016; Jo et al. 2014), smart education encourages the roots for a high-level use of technology, utilising it as a mind tool for creativity, collaboration and multimedia productivity (Gros 2016). According to Zhu et al. (2016), the goal of smart education is to foster workforce that masters twenty-first century knowledge and skills to address society's demands and challenges. 
In broad terms, smart education orbits learner centred approaches (Lister 2018; Gros 2016) with the aim of improving learner's quality of lifelong learning (Zhu et al. 2016). Zhu et al. (2016) emphasize that it focuses on contextual, personalized and seamless learning to promote learners' intelligence emerging and facilitate their problem-solving ability in smart environments. Despite smart education is increasing the demands for interactive and participative education content, Jo et al. (2014) observe there is a focus on infrastructure and smart devices rather than on educational effectiveness and efficiency. Such affirmation highlights the importance of building knowledge on the challenges the model should be confronted with, which can be associated with pedagogical theory, educational technology leadership and educational ideology (Zhu et al. 2016), for instance. In what regards pedagogy, Daniela (2019) establishes its growing importance within the process of adapting to smart education. According to this author, pedagogy becomes relevant because it is the tool which can actually unveil how technology should be applied meaningfully, i.e., technology cannot provide successful knowledge construction per se, however, it is an enriching tool.

Reflecting on the idea of smart associated with learning, Gros (2016) emphasizes the importance of technological design to make learning better, calling attention to its relationship to the term «technology-enhanced learning» (TEL). In that regard, Dron (2018) makes a provocative point, stating that the consideration of learners and teachers as active parts of environments provide no room for a not-smart learning environment. While contextualizing the term «smart» in regard to pedagogy, Daniela (2019) emphasizes its link with the development of Smart Technology, which underpins technology-enhanced environments. Still, and in line with Dron's (2018) conceptualization of «smart» as part of an ecosystem, Daniela (2019) provides a model of TEL in which both teachers and students play active roles, showing how determinant they are for the smartness arising in the knowledge building processes.

Considering that there is no single way of defining smart learning, Lister (2018) identifies a few commonalities in the discourse, including features as personalized learning, experiences enhanced by intelligent non-human agents, just-in time delivery of information and significance of geospatial relevance for content delivery selection. The same way, Zhu et al. (2016) list other key elements organizing smart learning as context-aware ubiquitous and tailored learning based on advanced IT infrastructure. Also, the author reinforces the interdependence between personal and smart technologies, approaching that learners engage in their learning in more open, connected and augmented ways by personally richer contexts. Hoel and Mason (2018) rely on a more basic and clear definition of smart learning, qualifying it as the challenging exploitation of smart environments for learning together with new technologies and approaches such as ubiquitous learning and mobile learning. Interestingly, Kearney et al. (2019) observe that smart learning characteristics are not entirely new, emphasizing that some of them such as collaboration and personalization have been actively promoted by some educators for many years with the aim of making learning more engaging, purposeful and meaningful. What has changed, according to these authors, is that both conditions and technologies are now aligning in ways that make these learning approaches more likely and achievable in comparison to the past.

For smart learning environments, one apprehends settings that emphasize learnercentric, personalized and adaptive learning service, interactive and collaborative tools, context-aware and ubiquitous access, with the aim of providing rich, personalized and seamless learning experience for learners (Zhu et al. 2016). Gros (2016) adds that a smart learning environment aims to support learners to obtain new knowledge, even while they are engaged in leisure activities while Hoel and Mason (2018) reinforce the model motto 
as being effective, efficient and engaging (see also Gros 2016; Zhu et al. 2016). According to Zhu et al. (2016), the goal of smart learning environment is to provide self-learning, self-motivated and personalized services which learners can attend courses at their own pace and are able to access the personalized learning content according to their personal difference. Clearly, personalization and adaptation are key factors in the provision of smart learning environments (Gros 2016), whilst these environments are meant to foster knowledge building processes through networks of interactions, meaning that the personalization in the smart learning framework does not stand for individual learning. On the very opposite, smart learning contexts motivates knowledge as an activation of individual cognitive competences to develop interactive and collaborative skills (see Lorenzo and Gallon 2019). It is a means to customise learning whilst still retaining the values and importance attached to social learning with peers (Kearney et al. 2019).

When reflecting upon smart learning environments, Dron (2018) distinguishes definitions as focused on tools, or occasionally on the techniques, that are found within them or of which they are constituted. According to this author, systems described as smart learning environments can be arranged into two general forms: (a) centralized models, which pervade the environment with an active role in both adapting to and shaping the behaviour of its inhabitants; and (b) distributed models, which put more emphasis on independent intelligent objects aimed at generating dynamics in mainly static environment. Dron (ibid.) explains that the centralized model focuses more on the orchestration of behaviour in the environment taken as a whole, while the distribute model is oriented towards augmenting the environment with smart components in a bottom-up, piecemeal manner. The author highlights that, usually, systems blend both models.

Prospecting on the benefits of smart education, Zhu et al. (2016) affirm that the smart learning environments could decrease learners' cognitive load, and thus enable learners to focus on sense making and facilitate ontology construction. For the authors, students' learning experience could be deepened and extended, and thus help students' development in an all-round way, i.e., affectively, intellectually, and physically. In this sense, Dron (2018) remarks that smartness of educational environments needs to be acknowledged as an emergent consequence of dynamic interactions between the environment's constituent parts, including those of its human inhabitants and the artefacts and structures they wittingly or unwittingly create, addressing the idea that every move in the overall environment has consequences on its level of perceived smartness.

Along with this critical overview on the implementation and experience of smart learning environments, Hoel and Mason (2018) assume that the more advanced the systems are, the more difficult it is to identify pedagogical practices, examples of technologies used and acknowledged standardization challenges. According to them, one explanation for this observation is that developing new technologies for learning and new practices is workin-progress. Pedagogy, as we see, assume crucial point in the building of smart scenarios, especially because, as observed by Dron (2018), digital environments only make up part of the learners' environment, which contains multiple intelligent inhabitants, who are themselves part of a shared environment, and who therefore constitute part of the environment for one another (see also Daniela 2019).

Based on the idea that a smart learning environment should actively provide the necessary learning guidance, hints, supportive tools or learning suggestions in the right place, at the right time and in the right form, Gros (2016) advocates for pedagogical strategies that feed conversation (conveying subjects and matters), reflection (including real time assessment based on students' progress and performance), innovation (mediated by breakthrough technology) and self-organization (including mechanisms of ongoing reorientation or 
resources). It is then reasonable that strategies take into consideration that within the richer and more precise conception of technologies as assemblies, not losing sight of the human within such assembly, pedagogies are displayed by different agents of education, rather than the teacher itself (Dron 2018). It is exactly such conception that allows the building of a learner-centred environment, in which, in the context of smart education, calls for the development of new thoughts about pedagogy based on existing theories that privilege the learner as an active asset in knowledge building (Gros 2016). In turn, Daniela (2019), advocating for the importance of a smart pedagogy, proposes that, from internal perspective, it might be the driving force of TEL, and from external perspective, it should ensure that for every activity there are three cornerstones to be taken into account: human developmental regularities (including conditions for the development of cognitive processes, sensory and socio-emotional threads), taxonomy of the educational process (including goals to be achieved) and technological progress (including teachers' pedagogical competence development).

Understanding that learners play a crucial role in the pedagogical process (Dron 2018), including in the smart education setting, and assuming pedagogy fit as a vital element for promoting behaviour change together with technology equipment (Yang et al. 2018), we might not take for granted, though, the role of teachers for the successful development of a smart learning environment (see Daniela 2019). At the end of the day, it is teachers themselves the strategic decision-makers that give dynamics to the curriculum and bring it live (Morgado 2017). A change in behaviour, therefore, a transformation in education setting, relies strongly on teachers' professional act, an object we explore further in the following section.

\subsection{Acknowledging Teachers as Drivers for Change in Education}

It is a common sense that we live in a world driven towards innovation, which is immediately related to technology assemblies (Tuomi 2006; OECD 2010; Thomas and Brown 2011). In this scenario, we observe that the characteristics of upbringing are changing, partly, due to the saturation of technology and information in our contemporary societies, imposing challenges both to society at large and to the educational field more specifically (Baruch and Erstad 2018). As we have demonstrated so far, regarding the idea of «smart» in education, ICT has clearly modified the conception of time and space, providing new opportunities to access information and arrange knowledge production (Gros 2016), even why traditional classroom arrangements became ineffective over time (Yang et al. 2018).

All over the globe, a relevant group of children and teenagers have easy access to personal computers and mobile technology, which is used with varied purposes. For that, school reality is pressed to change and adapt to these new realities, aiming at addressing students' abilities, interests and learning styles (Camilleri and Camilleri 2017). Zhu et al. (2016) observe that with the development of mobile, connected and personal technologies, for instance, mobile learning turned out as a major technology enhanced learning paradigm, with a further step being the reach of ubiquitous learning within the educational field. All these advancements in the educational context respond to what can be termed as the Fourth Industrial Revolution, marked by the convergence of physical, digital, and biological technologies that apparently change how or what people do and what it means to be human (Leahy et al. 2019; Schwab 2016).

Besides recognizing the change in education driven by students' abilities, interests and learning styles (Camilleri and Camilleri 2017), it is evident that educational transformation 
takes place since the twenty-first century skills clash with traditional schooling guided by standards (Baruch and Erstad 2018). Camilleri and Camilleri (2017) endorse that policymakers have quickly recognised the significance of certain technologies as a vehicle for socio-economic progress meanwhile stakeholders in education are also promoting innovative pedagogical practices by using technology. Moreover, Baruch and Erstad (2018) affirm that schools nowadays are more inclined to developing students as individuals and exploring directions such as creativity, as adjunct to ICT literacy to leverage lifelong skills that are important to future accommodation to the digital Era.

Bearing in mind the redefinition of educational setting through technology, in the context of futures studies, Leahy et al. (2019) argument that we need to consider the potentiality of new technologies and materials in education within the bricolage of factors impacting on peoples, planet, peace and prosperity, in a way that research considers learning opportunities across formal, non-formal and informal settings by reconnecting learning in schools with communities and families. Understanding this is a very immediate need, with the smart education perspective being one in progress, and recognizing that the use of technologies to facilitate learning and engage learners has become a universal phenomenon (Zhu et al. 2016), we then situate the place of teachers in such transformation.

Leahy et al. (2019) argue that the integration of new technologies in education is typically followed by a period of realization, identifying a gap between reality and expectations, in which a period where there is more critical consideration of the effectiveness or otherwise of the technology is settled. Gros (2016) adds that the use of technologies embedded within learners' habitual life experiences has important consequences for the pedagogical methods of formal education. Specifically, the author remarks that the inclusion of smart learning environments in educational contexts increases complexity as it demands education professionals to introduce innovative uses and new pedagogical approaches. For Leahy et al. (2019), such complexity also means trouble since the widespread integration of digital technologies in the classroom was followed by recurring issues of low teacher confidence and lack of adequate training for teachers, preventing a meaningful integration of technology in education (see also Earle 2002).

Assuming the relevance of teachers ageing in the Portuguese scenario, and in the rest of Europe (OECD 2019a), and contextualizing the complexities of enhancing smart education in compulsory formal education, we are interested in the role of veteran teachers (Admiraal et al. 2019; Carrilo and Flores 2018) concerning technology integration into classrooms. For «veteran», we consider teachers who have a vast experience in teaching, comprising a career over twenty years of practice. If we assume innovation as a process of change, we then realize teachers as the educational agents in the frontline for this move. There is a set of issues associated to changing curricular practices mediated by technology breakthrough, regarding the case of veteran teachers. Some of them relate to questions of tiredness or plateauing (Meister and Ahrens 2011), impacting on teaching disposition to incorporate change. Others comprise the digital gap status, which reveal a fragile familiarity of teachers with technology devices (Orlando 2014).

An extensive review of the literature about veteran teachers and the integration of technology in educational practice unveils two distinct mediators: (i) attitudes towards technology and (ii) sense making of pedagogical enhancement through technology resource (Louws et al. 2017; Chandra and Mills 2015; Shifflet and Weilbacher 2015; Lakkala and Ilomaki 2015; Orlando 2014; Buabeng-Andoh 2012; Meister and Ahrens 2011; Bingimlas 2009; Snoeyink and Ertmer 2001). Within these arguments, educational paradigms are referred as important departure points in terms of teachers' disposition to elaborate new learning environments, with identification of a more positive response from those aligned 
with constructivist theories (Lakkala and Ilomaki 2015; Chandra and Mills 2015; Orlando 2014; Snoeyink and Ertmer 2001).

In terms of teachers' attitudes, despite considerable research suggests that positive mindset regarding technology promotes the adhesion to related practices in education (Chandra and Mills 2015; Bingimlas 2009), there are also records of mismatches between positive attitude and actual practice transformation (Camilleri and Camilleri 2017; Shifflet and Weilbacher 2015; Buabeng-Andoh 2012). Mainly, such mismatches are interpreted in relation to issues associated with different dimensions of change in the educational field. Orlando (2014) highlights that along their careers, teachers are constantly called for participating in practice change and that, at many times, they are not consulted on designing these changes, thus generating a sense of uninterest for an educational move.

It is unquestionable though that teachers' competence and confidence in manipulating technology is a key factor in incorporating it into teaching (Khlaif 2018; Lakkala and Ilomaki 2015; Chandra and Mills 2015; Bingimlas 2009), and as so, training emerges a decisive variable for successful change in teaching culture (Camilleri and Camilleri 2017). According to Louws et al. (2017), there is high interest by the educational community in technology training, leading to the idea that what matters is identifying what level of competence teachers have in order to establish appropriate training (Snoeyink and Ertmer 2001). As Camilleri and Camilleri (2017) sustain, increasing professional development opportunities for teachers is an efficient way of boosting ICT engagement in teaching and learning, because it helps build confident and supportive teachers.

Acknowledging this important place teachers secure in the development of technologyenhanced learning, thus, in the ongoing implementation of smart education-or would it rather be smarter? -, in the next sections, we draw on a training focused on mobile learning and related activities to ascertain a debate on how teacher training can be determinant for a digital move and the incorporation of new practices in schooling. Initially, we establish our research questions, provide the grounds of the empirical work sustaining this article, contextualize the training and its participants and then, we propose an analysis of the training model efficiency and teachers' perceptions of it. At a final stage, we shed light on how the struggle to promote digital migration of veteran teachers benefit the ongoing culture towards a smart education scenario.

\section{Methodology}

Admitting that the ecosystem of technology-enhanced learning, as in smart education, consists of a combination of different elements from which pedagogy is a key factor (Daniela 2019; Dron 2018; Hoel and Mason 2018), we seek to comprehend in depth what aspects provide efficiency for digital migration in formal teacher (in-service) training. By digital migration, we mean not only the acquisition of technology competences, but also the capability of reading it into a pedagogical perspective (Daniela 2019; Earle 2002). As we understand, building a smart education environment involves a period of realization, confronting both reality and expectations (Leahy et al. 2019), and as such, teacher training makes up a fertile ground to exploit efficiency clues for rooting culture change. In this sense, and having as targeted group veteran teachers, we address two issues within this article:

(a) What are the reasons why veteran teachers care for technology-oriented training and are they willing to move into smart(er) education scenarios? 
(b) What key aspects underlies a successful technology-oriented training for veteran teachers?

\subsection{Context: Rekindle+50 training}

The training initiative, which underpins this study, was assembled by two education sciences faculties in Portugal in association with two teachers' training centres from central and northern areas of the country, which are actively involved in the "Future Classrooms" program. This latter program, also known as Innovative Educational Environments, aims to address issues of student demotivation and school achievement by promoting new methodologies in teaching. For that, training centres are equipped with updated technology devices.

The action Rekindle +50 focuses on teachers over 50 years old and on renewing their commitment to teaching and curricular innovation through the deployment of mobile technologies in educational context. Acknowledging that Portuguese teacher force has undergone accelerated process of ageing (OECD 2019b), the project was designed as an attempt to empower their long-term trajectories with digital knowledge. In broad terms, the action was designed to bridge the gap between veteran teachers (Carrilo and Flores 2018; Orlando 2014) interests and the digital culture, arranging room for interconnectivity around teachers and students' alterities, usually set apart, strengthening a common ground through technology language.

The project links research to intervention and to pre-school, basic and secondary school teachers' training. Rekindle +50 workplan involves diagnosing, monitoring and evaluating the change in practices, within a movement on digital migrations, with focus on the possible sustainability of this move in the short and medium term. Along 2019, thirty-eight teachers of different subject domains from all levels of compulsory education (in Portugal, it includes upper secondary level) and pre-school participated in educational actions under the project reference.

\subsection{The Participants}

Initially, thirty-eight teachers were enrolled in the Rekindle +50 training on mobile technology at both teachers' training centres, in Maia (Northern Portugal) and Mangualde (Central Portugal). After a few dropouts and consequently replacements, 34 teachers completed the program. All teachers signed up for the course were 50 years old or older, except for a male Mathematics teacher. Profile of participants does not consider his answers to the questionnaire $(\mathrm{N}=32$, due to one teacher not responding the questionnaire). The group average age was 55, being the oldest professional a 62-year-old Physics and Chemistry teacher and the youngest one a 50-year-old Biology teacher. Both are women. Age distribution is detailed below in Fig. 1.

One appealing aspect of the group regards the number of language teachers, a total of 15 participants, ranging from Portuguese to French and English content-areas. It is also expressive the participation of 10 teachers from disciplines such as Mathematics, Physics or Chemistry. In Mangualde, it was distinctive the participation of two female teachers coming from pre-school setting. If we isolate disciplines, Portuguese teachers make up the majority, seven of them exclusively leading this content-area and other four being responsible for other disciplines as well, such as English, History or Geography. Physics and Chemistry area is the origin of other six teachers. Figure 2 details the content-area distribution. 


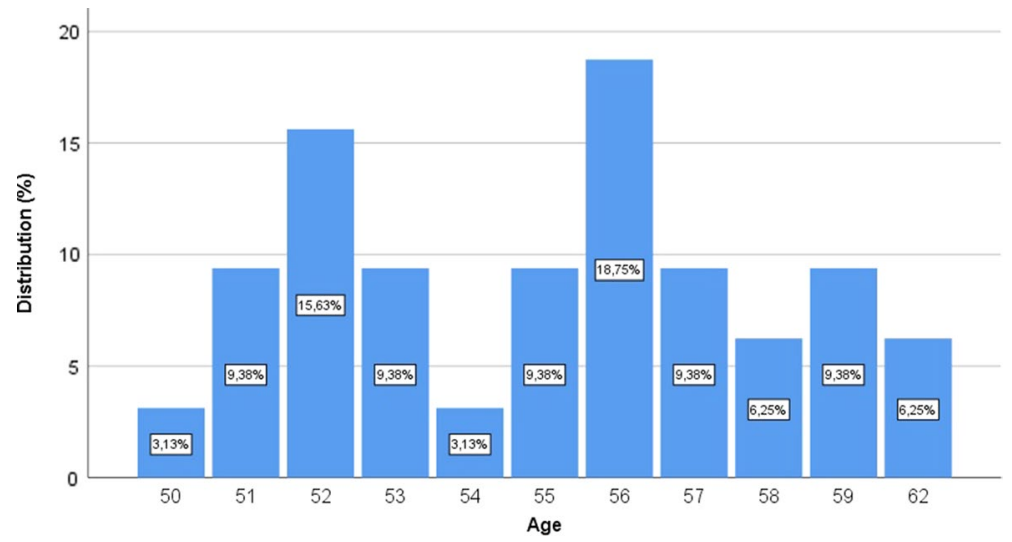

Fig. 1 Teachers' age distribution

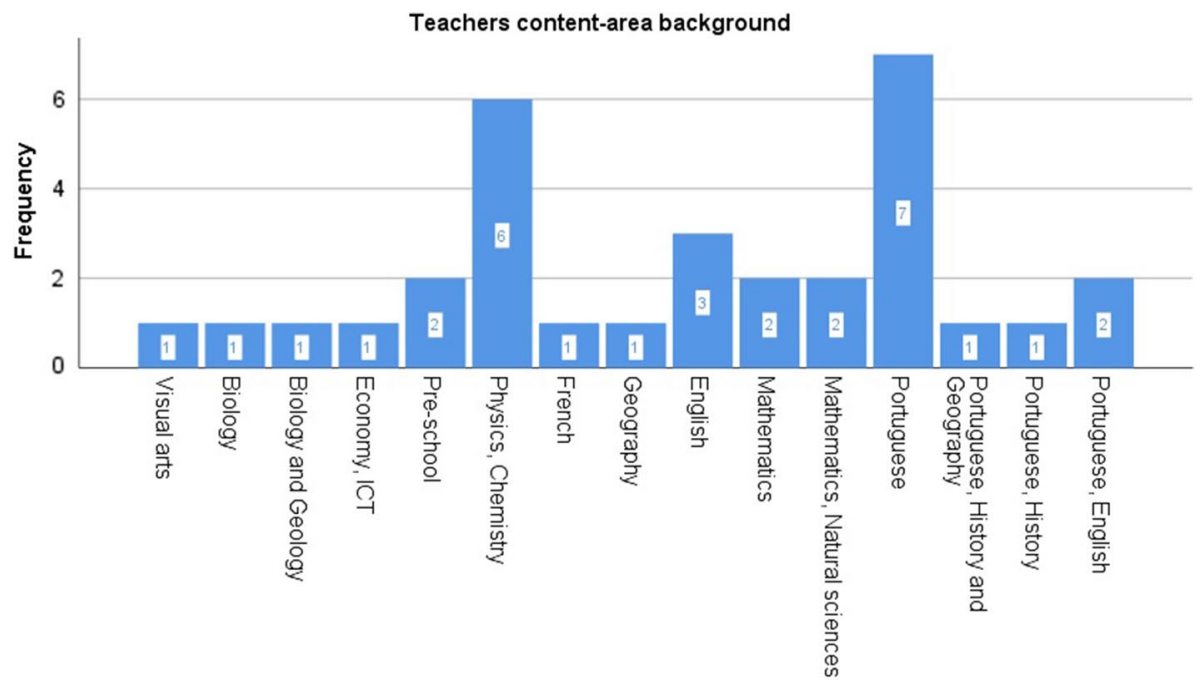

Fig. 2 Teachers' content-area background

In what concerns the professional experience of the participants, we have an average of 29 years of teaching practice (4 teachers did not answer this question), reinforcing the idea that audience is made up of veteran teachers (Carrilo and Flores 2018; Orlando 2014). Confirming this nature of the participants is key to understand not only their needs in terms of technology driven pedagogy, but also regarding the kind of training that is more suitable to their professional development (Lakkala and Ilomaki 2015; Bingimlas 2009). Should we not forget one of the goals of the training is to rekindle careers that might have reached a plateau stage (Meister and Ahrens 2011) for example, due to very different reasons-being it intergenerational barriers, institutional disenchantment or political disbelief/tiredness. Among the participants, the less experienced teacher holds a trajectory of twenty-one years in teaching, whereas the most experienced one is currently on her fortieth year in formal education-see Table 1. 
Table 1 Teachers' professional experience

\begin{tabular}{lll}
\hline Teachers' professional experience (years) & \\
\hline $\mathrm{N}$ & Valid & 28 \\
& Missing & 4 \\
Average & 29.5 & \\
Minimum & 20 & \\
Maximum & 39 & \\
\hline
\end{tabular}

Another aspect of interest concerning the participants' profile of the training on mobile technology is their involvement in activities of pedagogical leadership at their institutions. Only eleven out of thirty-two respondents declared some sort of function on these grounds, suggesting that such element does not configure a strong factor of adhesion to the program. Among these eleven teachers, the functions range from Project Coordinator to Principal's Assistant. Other activities concern Teachers' Supervising, Distance Education Coordination and VET Leadership.

Apart from pedagogical leadership activities, seven teachers are Class Leaders, a function which is institutionalized in Portugal. Every class has an appointed Class Leader, which is a teacher in charge of mediating the communication between school and parents and, more importantly, following pedagogical development of students. At total, eleven participants strictly teach in their respective schools. An overview of the duties profile leads to the understanding that changing technology culture at schools can still be a matter of individual effort, assuming that staff in more strategic positions seem not to be significantly involved in training of this nature. It is noteworthy that

Table 2 Professional duties

\begin{tabular}{lll}
\hline Professional duties & & \\
\hline N & Valid & 32 \\
& Missing & 0 \\
Nature of duties & Frequency & Percentage $(\%)$ \\
Tutoring teacher & 1 & 3.1 \\
Teacher; Teacher of Distance Secondary Education for Adults Program; & 1 & 3.1 \\
Scientific and didactic consultant for STEM project & & \\
Teacher; Coordinator of Distance Secondary Education for Adults Program & 1 & 3.1 \\
Teacher & 11 & 34.4 \\
Teacher; Class leader & 7 & 21.9 \\
Teacher; Principal assistant & 3 & 9,4 \\
Teacher; 7th Grade Pedagogical team coordinator & 1 & 3.1 \\
Teacher; Coordinator of VET on Health & 1 & 3.1 \\
Teacher; Class Leader; ICT Support; Electrician & 1 & 3.1 \\
Teacher; Class leader; Class leaders coordinator & 2 & 6.3 \\
Teacher; Infrastructure manager & 1 & 3.1 \\
Teacher; Adult Teaching Unit coordinator & 1 & 3.1 \\
Teacher; Project and Activity Board coordinator & 1 & 3.1 \\
Total & 32 & 100 \\
\hline
\end{tabular}


only one teacher has background duties associated with ICT tasks. Table 2 provides a detailed description of participants' professional duties.

\subsection{Instruments, Data Collection and Analysis}

In order to address our research questions, we designed an online questionnaire to collect teachers' conceptions on mobile technology as well as their level of interaction with related devices. Also, the questionnaire envisioned to outline a profile of participants (as presented above). The instrument was, therefore, organized into three sections. The first one covered profile related questions (age, gender, teaching experience, content-area expertise and general responsibilities at school level). In a second segment, the questionnaire comprised 10 items regarding teachers' use and beliefs concerning mobile technology. This section assembled Likert-type questions and included items such as:

- I usually use my mobile phone to manage professional tasks

- I understand mobile technology as a pedagogical resource that should be exploited

- I think mobile technology should be more regularly applied as a resource for teaching/learning activities.

Finally, a third part of the questionnaire comprised two open questions in regard to teachers' beliefs on potentialities and risks of resourcing on mobile technology in classrooms activities.

Apart from the questionnaire, we also relied on teachers' digital learning diaries, which were held on Padlet platform by all participants. These diaries were mandatory for teachers registered in the training and intended to encourage participants to reflect on their daily progression. Each week, participants would insert evidence on their tasks outputs. Moreover, they were encouraged to make entries reflecting on their learning journey in the scope of TEL, which included both initial expectations at the beginning of the training and a thorough evaluation of the process at the end of the programme. As previously mentioned, from thirty-eight teachers initially registered for the training in both centres, thirty-four attended the action until its completion. Of those, thirtythree answered the questionnaire, which was distributed in the beginning of the action. When it comes to the learning diaries, all thirty-four Padlets were targeted for analysis.

Content (emergent category-driven) and descriptive analysis (Pereira 2010; Freires and Pereira 2018, Freires et al. 2019) of both questionnaires and learning diaries were performed arranging information in the following dimensions: teachers' perception of technology (acceptance or refusal), digital competence (usability routine), pedagogical beliefs (in regard to technology integration) and training expectations (purposes for registering). The results of the analyses together with a thorough reflection on the training syllabus constitute the backbone of forthcoming discussion.

All teachers involved in the project consented the use of collected data for research purposes. The citations throughout the text refer to fictitious names, addressing the commitment to confidentiality and anonymity, in accordance with ethical guidelines (Freires and Pereira 2020). 


\section{Designing a Training Model: Key Observations}

Bearing in mind the teachers' profile and the goal of rekindling teaching through technology-enhanced education, in the framework of smart learning environments, we now elaborate on the designing of the training offered by the project. The training was arranged in nine sessions and lasted $50 \mathrm{~h}: 25 \mathrm{~h}$ face-to-face and $25 \mathrm{~h}$ of autonomous work, in which teachers worked with their students in real educational settings. This design was intended to assign a regularity between the sessions and allow the teachers' practical experimentation with their students. We established the following Learning Outcomes:

- To develop pedagogical skills to use mobile devices in the educational context;

- To promote the use of mobile devices by teachers and students;

- To reflect on Innovative Learning Scenarios;

- To design new environments in learning spaces;

- To encourage the application of innovative pedagogical models such as Flipped Learning, Gamification, Game-Based Learning and Digital Storytelling with the use of mobile devices;

- To design activities considering the proposed pedagogical approaches;

- To implement planned activities using the proposed pedagogical approaches;

- To create digital resources for the use of mobile devices in educational contexts.

All contents and resources were available online in the course platform since the beginning of the training, offering teachers the opportunity to experience content freely and extend the concept of training to anywhere at any time, providing them an opportunity to live the seamless character (Zhu et al. 2016) intended in the framework of smart education.

Initially, the course focused on the introduction of mobile learning, its definition, characteristics and potentialities in the educational scenario. Learning spaces were also a hot topic through the exploitation of links between learning goals and space design. Content discussions resourced on a variety of mobile applications, such as quizzes, collaborative writing, digital writing, augmented reality, 3D, video storytelling, mental maps, interactive content platforms, web tools and gamification platforms. These tools served the purpose of exploring creativity, critical thinking, autonomy, as well as cooperative and collaborative work.

Considering that teachers participated in training after a hard day's work, we tried to involve them within a gamification strategy. The gamification was used with the teachers in a model to be replicated with their students. We drew a narrative collaboratively for the training course, where each teacher could be a character in a treasure hunt story with a map of conquests. We attributed (Seaborn and Fels 2015): (i) Points (numerical units indicating progress), (ii) Badges (visual icons signifying achievements), (iii) Leaderboard (display of ranks for comparison), (iv) Progression (milestones indicating progress), (v) Status (textual monikers indicating progress), (vi) Levels (increasing difficulty tasks), (vii) Rewards (tangible, desirable items), and Roles (role-playing elements of character). All these game elements supported the fulfilment of the training course goals.

For each topic covered in each training session, teachers developed, through peer collaboration processes, pedagogical resources and a lesson plan. Between sessions, each teacher individually applied the lesson plan with their students. In the following training session, the first moment comprised a collective reflection among colleagues, with a step of writing down the experiences shared. Task 4, for instance, required teachers to develop 
videos in order to lead a class in the flipped learning model (see Lencastre et al. 2020). Teachers were exposed to storyboard planning and applications that would ease the audiovisual component assembly during the training. For one of the participants, an English teacher, it resulted on the production of a series of videos on "Types of house" with elementary level students. She delivered a release video on the subject and guided students to exploit content by themselves. In a following stage, divided into groups, students were invited to develop their own videos and share results on new knowledge with the entire class. Another popular activity was task 12 , in which teachers were challenged to elaborate a quiz (online), resourcing not only on text, but also on interactive content. In this case, a Geometry teacher applied the concept to review content before a test. He found it particularly useful due to resources of augmented reality that allowed the in-depth exploitation of geometrical shapes.

Besides the formal training, we held non-formal follow-up sessions in situ to consolidate and reinforce competencies, to contribute to teachers' greater confidence in the application of innovative pedagogical approaches - some participants aligned their age to a lower confidence in technology resource. That meant one researcher was available to visit teachers in their own schools while performing lessons on environments driven by technology. After each class observation, researcher and teacher would have a conversation to pinpoint aspects explored during the lesson. It was thought to be a moment of joint reflection in the process of digital migration.

Another valuable aspect of the training design was the participation of special guests, usually academics, whose participation was driven to promote debates on key areas of the course. Each training module involved three guests, specialists working on curriculum, professional identity and ageing. They would usually occupy half of a session and their lecturers were planned to integrate teachers' view on a critical analysis of contemporary society

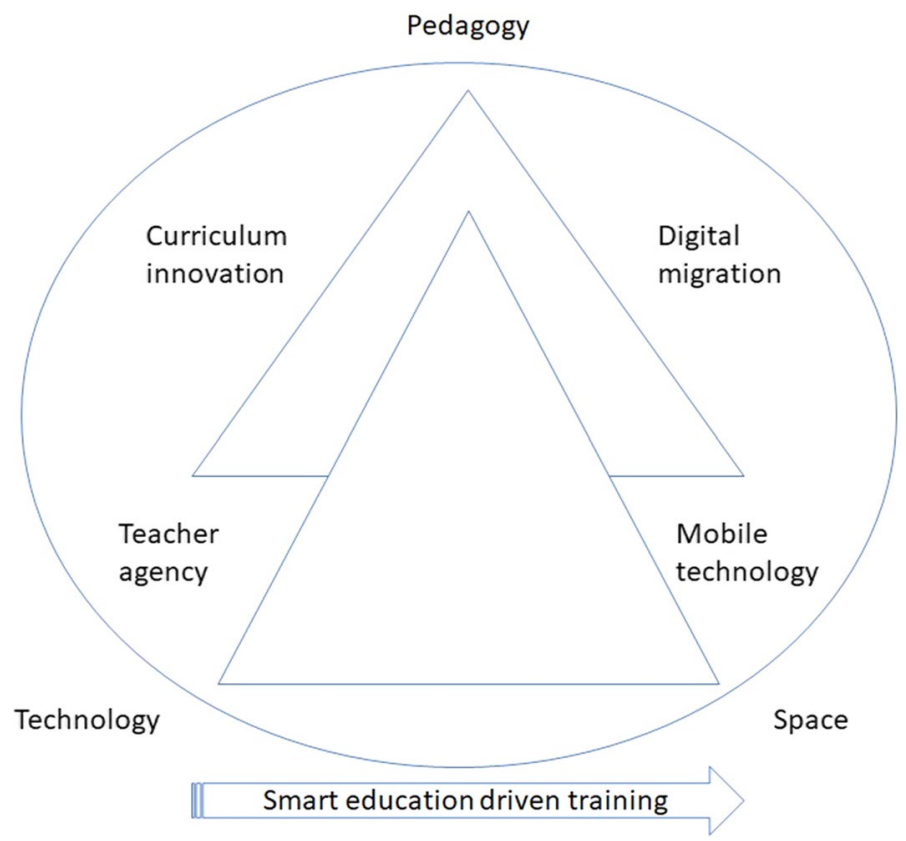

Fig. 3 Rekindle+50 training design 
educational setting. As it might be clear, the issues in focus were keen to the participants' profile. Below, Fig. 3 depicts a visual representation of the training key components.

Focusing on our second research question, in terms of aspects that underlie an effective technology-oriented training for veteran teachers, we might highlight three driving forces: profile design, personal guidance and continuing follow-up. Understanding teachers' profile is basic to technology training (Lakkala and Ilomaki 2015; Bingimlas 2009) as it is the departing point to increasingly equip teachers to foster links between technology and pedagogy. Bearing in mind their perceptions and resourcing on them support developing capabilities more efficiently. Likewise, it remains true that teachers strive to make a move in pedagogical innovation in isolated ways. Most of the participants were the only attendees from their schools or had a couple of colleagues. This requires an added dimension of guidance since literature points out that change works better when implemented in and by networks (Meister and Ahrens 2011). And at last, and because of the isolation feature, continuing follow-up turns out to be consistently motivating. Not all teachers opted for having the guest researcher in their classes, but those who have, reflected on how it encouraged them both to plan and to execute classes in technology rich environments. It also encompassed a sense of technical support (Bingimlas 2009), despite this dimension not being the focus of the visit. As we see, it allowed teachers to pave new avenues in a moment of realization (Leahy et al. 2019). The follow-up was also remarkable in acknowledging that schools' infrastructure limit opportunities for teachers to feed smart(er) education environments.

\subsection{Advancing to the Frontline: Teachers Willing to Change}

It is well established in the literature that teachers' perceptions and attitudes towards technology and their sense making of pedagogical enhancement by technology deployment directly interfere in their willingness to implement change in coursework (e.g. Lakkala and Ilomaki 2015). In the specific case of veteran teachers, the challenge into adapting to a more digital world have increased barriers like the feeling of tiredness or frustration for continuing imposed change (Orlando 2014). Nonetheless, the involvement of teachers in actions like the Portuguese training in analysis demonstrate they are neither throwing the towel nor running behind in what regards society's current digital perspective-although they are just crawling yet.

The questionnaires and digital learning diaries' analyses provides three main reasons why teachers care for advancing into a smarter education scenario, helping systematize our answer to research question one. In the first place, the changing perspective is fed by a sense of professional development. As stated by Lea "Stopping is dying", referring to the idea that ignoring innovation in pedagogy relates to losing the thirst for performing the job itself. This dimension is closely related to the sphere of pedagogical relationship, a second main reason why teachers seek to integrate technology in their classrooms. It implies the expertise of new techniques, the increase of classes attractiveness and it even contains an element of fighting against indiscipline. In this regard, Alice explains that she seeks for technology training because "I want to learn again how to apply mobile technology that supports teaching the syllabus in a more interesting and exciting way". Finally, a third reason links with consolidating an improved relationship with students, with teachers being able to grasp a bit more of their personal interests in which technology applies. While placing students as drivers for personal change, teachers tacitly respond to a growing culture of learner centred approaches (Daniela 2019; Lister 2018; Gros 2016) in schooling. 
Accordingly, Rita justifies her enrolment in the training since she wants to "provide students with more interesting learning to respond to the digital era of which they are part of".

In what concerns fears or constraints, possibly leading to what Daniela (2019) refers to as a centrifugal effect, i.e., the inappropriate detachment of technology from pedagogy, teachers participating in the training mostly affirmed a perceived lack of control regarding media browsing. They are afraid technology driven environments lead students to navigate on inappropriate websites or drive them away of content interest-what reinforces the role of a smart pedagogy (see Daniela 2019). This assumption reveals the importance of developing smart systems at school level—networks are important for implementing innovation (Bingimlas 2009) — while also denounces teachers' lack of familiarity with technical implications. In general, schools' networks are equipped with systems that avoid students to having access to full open navigation. A curious aspect regarding the move towards smarter education is the fear of exposure. A male teacher refers that he feels uncomfortable that his activities might be recorded and shared online.

\subsection{Digital Migration of Veteran Teachers: Is there Room for Innovation?}

Considering the tailored training presented previously, as well as teachers' thoughts on it, we now provide a few considerations on its potentiality towards integrating veteran teachers into the smart education framework. Mainly, we weave our arguments in regard to key aspects such as teachers' profile, resources availability and culture perspectives. Data that sustain this analysis consists of continuing debate on the training model, content and descriptive analysis of questionnaires applied to teachers and their digital learning diaries.

To start with, as we have widely exposed, this training was aimed at veteran teachers. Literature suggests that appropriate training, if meant to be successful, has to address the trainees' profile and their respective needs (Lakkala and Ilomaki 2015; Snoeyink and Ertmer 2001). As observed in situ, age was never the trouble for teachers enrolled in the course, actually, from their own perspective, holding a long term career increases their ability to design curriculum in innovative ways, due to the expertise they have established along the years. Yet, digital competencies are an obstacle in the scenario of a smart education framework, not because teachers do not follow the devices solely, but because they struggle to apply them with a pedagogical purpose. There is a road to be taken, and understanding the specifics of this road is what turns the training into an asset, when it reads what kind of knowledge is desired by teachers, something that also is reached by provoking them-through debating issues like ageing and technology enhancement.

As explored, a very important aspect of the training is highlighted on its offer to visit teachers at their own schools. Peer work was also encouraged, meaning teachers from the same school clusters could observe each other. Curiously, the few that would have such opportunity sustained a view that they were not able to perform such task, assuming a lack of expertise in technological aspects, despite the previous preparation encouraged during the training. It is also remarkable that from all participants, only nine ended up adhering to this collaboration. In here, there is a clear sense of «invasion» or «trespassing» regarding teachers' natural habitat. Most teachers feel uncomfortable with the possibility of being observed, even though the explicit agreement was of partnership and not assessment. As Lakkala and Ilomaki (2015) and Bingimlas (2009) affirm, when it comes to technology, teachers do not want to be exposed or embarrassed through their teaching choices. For those who took a chance, though, the training reached a deeper level with the possibility of them taking the stage and leading part of some sessions to share their achievements. Of 
course, the ones who engaged in this partnership were those who had more familiarity with pedagogical employment of technology, therefore, willing to take more risks. On a footnote, the visiting researcher observes that infrastructure is sometimes an issue at schools. In some of them, the smart education scenario is just not a possibility, although some technology-enhanced learning might be driven. As we defend in this article, such a step can encompass an initial direction into smarter educational settings, and that matters.

In conformity to the program design, all teachers were expected to apply new learning to practical activities with their students between sessions. A considerable number of trainees failed to fulfil all tasks. The reasons presented for that behaviour consist mainly of a matter of time or lack of infrastructure, as informed. Apparently, this attitude does not encompass a mood of uninterest or lack of digital competencies on behalf of teachers (Orlando 2014). In terms of content-area, it is remarkable that Mathematics teachers would make a point on missing the link between technology-driven activities and their subjects. In comparison to language and other humanities content-area, these teachers revealed struggling more to adapt to a technology driven learning environment, what we read in terms of not identifying clearly the potential links of pedagogy and technology (Daniela 2019; Earle 2002). When activities were invested in more concrete, «palpable» formats, the inversed perspective would take place. Science teachers, for example, found augmented reality tools quite enlightening and rapidly conceived a pedagogical plan for implementing it, as digital diaries revealed. Regarding the infrastructure issues, teachers drew attention to weak Internet connection or lack of equipment-reduced number of computers, tablets, etc. In more specific cases, students economically deprived were pointed out as reasons to avoid practical experiences in classrooms - they would even lack mobile phones.

Globally analysing the feedback of teachers and their development on establishing technology enhanced practices, one understands the high impact of institutional culture on innovative transformation of teaching (Orlando 2014; Bingimlas 2009). For those who were the only ones from their schools taking the training, incorporating new practices was clearly a bigger challenge. The sense of isolation was also identified as a demotivation factor, as we registered before.

Overall, the analysis of the training model unveils a strong dimension of human interaction as a sustainable driver for change. It implies movements of support, dialogue, risk taking and networking. In accordance with Dron (2018) observations, the smart in education encompasses a good deal of human elements. In this sense, the close follow-up of teachers' development and the collaboration within school classes, although seemed frightening to some, proved to be very meaningful and exciting, prompting teachers to defy more their own practices and redesign curriculum. As we have thoroughly explored throughout the training, teachers are and have to think of themselves as strategic curriculum decision makers (Morgado 2017).

Thinking strictly of what smart means in a smart education framework, we would claim our action as a moderate effort to engage teachers within different intelligence levels of varied smart systems (see Hoel and Mason 2018). Assuming it is a very initial step towards a new teacher culture for most of the teachers involved, we still infer that the course touches practically all driving forces of different smartness levels, as proposed by Hoel and Mason (2018), namely, adapt, sense, infer, learn, anticipate and self-organize. Moreover, it is in line with basic assumptions of smart learning environments, including perspectives on learner-centric, personalized and adaptive learning service, interactive and collaborative tools (Kearney et al. 2019; Zhu et al. 2016). From data analysis, we understand that a few features need to be adjusted to meet a more comprehensive sense of a learning experience which is both seamless and engaging (Lorenzo and Gallon 2019; Gros 2016; Zhu et al. 
2016). As we understand, this move also depends on a better alliance with schools and their capability to foster tools availability.

On empirical grounds, one can almost concretely touch the realization phase (Leahy et al. 2019) in action. Of course, not all teachers have access to smart systems, but they end up the training prepared to start dealing and growing themselves the level of smartness of their learning environments. In a few words, a digital migration has to be seen as a continuous process. Valuing this process for those who have already become a veteran teacher is an asset in a country where more than fifty per cent of all teaching force has surpassed the age of 50. And most differently from what common sense could nurture, veteran teachers are far from being unable to manipulate technology - their perceptions of it have a deeper impact on practice (Khlaif 2018; Lakkala and Ilomaki 2015; Chandra and Mills 2015). Their answers to the questionnaires and digital diaries reflect both their interest and thirst to keep improving.

\section{Conclusions}

We live in a technology driven world, in the context of a Fourth Industrial Revolution, and this is irreversible. On these grounds, it is of ultimate importance that education embraces this revolution if we aim to prepare students for contemporary societies. Among huge challenges education has to deal with, veteran teachers - and not only — and their capability of adjusting to this new Era should be a formal commitment, especially in countries like Portugal, where the ageing of the teaching force is massive.

Shaping the Fourth Industrial Revolution on in-service training to ensure that it is empowering and student-centred, rather than teacher-centred, is the task for any single stakeholder on the educational sector. It is, therefore, imperative that we provide attention to in-service training, enabling veteran teachers to participate in, and benefit from, the ongoing transformations.

The training we designed and our effort to reach this smarter education scenario proves that a long path is yet to be taken. Of course, this is a mainly qualitative oriented research with a very restricted number of teachers. Although results cannot be generalized and represent, partially, specificities of the Portuguese context, its reading in connection with related research in the field sheds some light on the ongoing transformation of educational practices, concerning the arising digital world. Explicitly, it allows us to reach some clues on how to perform change in a more sustainable way. In the first place, we have to leave behind the myth that older teachers cannot handle technology. Actually, as pedagogues, we might embrace them in a joint effort to realize how technology and pedagogy can be combined in meaningful ways. It is important to prompt new experiences and create comfortable and safe places for experimentation. Schools have an important responsibility in here. Realizing that not all teachers have got access to the infrastructure necessary to perform technology enhanced activities does not erase the fact that, otherwise, most teachers have enough basics to start. Equally, it is necessary to find out ways to build networks of innovative practices that go beyond one school's walls. When teachers are «breakers» in their institutions, the training group can occupy that place of collegiality.

An essential key in fulfilling the digital migration is seeing it as a collaborative and continuous practice. Such conception empowers teachers and redesign the relationships they establish with their own students. No training can be fruitful if teachers' profile, reality and context are not taken into consideration. A twenty-first century society will only benefit its 
students when it accurately addresses the reality of its teachers. In other words, it is a not a top down revolution, but a participated one. Tailored profiled training, close follow-up support and curriculum decision make encouragement are vital elements in this process. A hard one, because in progress.

In global terms, the present study then helps localizing a twofold approach for the fostering of smart education provision. On the one hand, in-service training, pedagogicallydriven, emerges a pre-condition if technology integration intends to be meaningful. On the other hand, collegiality must be pursued as networks on knowledge building proves to allow a sense of sustainability over time. Due to our study limitations (number and origin of participants, limited practices' follow-up), the debate would benefit from further research intertwining the veteran profile with the resource on TEL. It would be significant to explore specifics of profile, such as content-area expertise, gender and professional experience record. Longitudinal research on this theme (quite rare, but which we are committed to conducting) would most definitely be of ultimate guidance to the discussion as well.

Acknowledgements This article was developed within the project Digital migrations and curricular innovation: giving new meaning to experience and rekindling teaching profession after 50, funded by the Portuguese Foundation for Science and Technology (FCT), under the grant PTDC/CED-EDG/28017/2017.

\section{References}

Admiraal, W., Veldman, I., Mainhard, T., \& Tartwijk, J. (2019). A typology of veteran teachers' job satisfaction: Their relationships with their students and the nature of their work. Social Psychology of Education: An International Journal. https://doi.org/10.1007/s11218-018-09477-z.

Baruch, A. F., \& Erstad, O. (2018). Upbringing in a digital world: Opportunities and possibilities. Technology, Knowledge and Learning. https://doi.org/10.1007/s10758-018-9386-8.

Bingimlas, K. A. (2009). Barriers to the Successful Integration of ICT in teaching and learning environments: A review of the literature. Eurasia Journal of Mathematics, Science and Technology Education. https://doi.org/10.12973/ejmste/75275.

Buabeng-Andoh, C. (2012). An exploration of teachers' skills, perceptions and practices of ICT in teaching and learning in the Ghanaian second-cycle schools. Contemporary Educational Technology. https:// doi.org/10.30935/cedtech/6066.

Camilleri, M. A., \& Camilleri, A. C. (2017). Digital learning resources and ubiquitous technologies in education. Technology, Knowledge and Learning. https://doi.org/10.1007/s10758-016-9287-7.

Carrilo, C., \& Flores, M. A. (2018). Veteran teachers' identity: what does the research literature tell us? Cambridge Journal of Education. https://doi.org/10.1080/0305764X.2017.1394982.

Chandra, V., \& Mills, K. A. (2015). Transforming the core business of teaching and learning in classrooms through ICT. Technology, Pedagogy and Education. https://doi.org/10.1080/1475939X.2014.975737.

Daniela, L. (2019). Smart pedagogy for technology-enhanced learning. In L. Daniela (Ed.), Didactics of smart pedagogy. Smart pedagogy for technology enhanced learning (pp. 3-22). Cham: Springer.

Dron, J. (2018). Smart learning environments, and not so smart learning environments: A systems view. Smart Learning Environments. https://doi.org/10.1186/s40561-018-0075-9.

Freires, T., \& Pereira, F. (2018). Secondary specialist arts education: Perceptions of youths in Northern Portugal. Educação e Pesquisa. https://doi.org/10.1590/s1678-4634201707160053.

Freires, T., \& Pereira, F. (2020). Por uma investigação eticamente responsável: reflexões a partir do trabalho narrativo em educação [For an ethically-driven research: reflections based on the narrative work in education]. In P. Savi Neto \& I. Nascimento (Eds.), Ensaios sobre ética e investigação em psicologia e em educação [Essays on ethics and research in psychology and education] (pp. 61-71). Porto: Legis.

Freires, T., Bento, M., Morgado, J. C., \& Lencastre, J. A. (2019). Professores veteranos e inovação curricular: desafios do recurso à tecnologia como instrumento pedagógico [Veteran teachers and curricular innovation: challenges of deploying technology as a pedagogical tool]. In C. Leite \& P. Fernandes (Coords.), Currículo, avaliação, formação e tecnologias educativas (CAFTe). Contributos teóricos 
e práticos [Curriculum, evaluation, training and educational technologies (CAFTe). Theoretical and practical contributions] (pp. 761-772). Porto: CIIE.

Earle, R. S. (2002). The integration of instructional technology into public education: Promises and challenges. ET Magazine, 42(1), 5-13.

Gros, B. (2016). The design of smart educational environments. Smart Learning Environments. https://doi. org/10.1186/s40561-016-0039-x.

Hoel, T., \& Mason, J. (2018). Standards for smart education-towards a development framework. Smart Learning Environments. https://doi.org/10.1186/s40561-018-0052-3.

Jo, J., Park, K., Lee, D., \& Lim, H. (2014). An integrated teaching and learning assistance system meeting requirements for smart education. Wireless Personal Communication. https://doi.org/10.1007/s1127 7-014-1765-4.

Kearney, M., Burden, K., \& Schuck, S. (2019). Disrupting education using smart mobile pedagogies. In L. Daniela (Ed.), Didactics of smart pedagogy. Smart pedagogy for technology enhanced learning (pp. 139-158). Cham: Springer.

Khlaif, Z. N. (2018). Factors influencing teachers' attitudes toward mobile technology integration in K-12. Technology, Knowledge and Learning. https://doi.org/10.1007/s10758-017-9311-6.

Lakkala, M., \& Ilomaki, L. (2015). A case study of developing ICT-supported pedagogy through a collegial practice transfer process. Computers \& Education. https://doi.org/10.1016/j.compedu.2015.09.001.

Leahy, S. M., Holland, C., \& Ward, F. (2019). The digital frontier: Envisioning future technologies impact on the classroom. Futures. https://doi.org/10.1016/j.futures.2019.04.009.

Lencastre, J. A., Morgado, J. C., Freires, T., \& Bento, M. (2020). A systematic review on the flipped classroom model as a promoter of curriculum innovation. International Journal of Instruction. https://doi. org/10.29333/iji.2020.13436a.

Lister, P. (2018). A smarter knowledge commons for smart learning. Smart Learning Environments. https:// doi.org/10.1186/s40561-018-0056-z.

Lorenzo, N., \& Gallon, R. (2019). Smart pedagogy for smart learning. In L. Daniela (Ed.), Didactics of smart pedagogy. Smart pedagogy for technology enhanced learning (pp. 41-70). Cham: Springer.

Louws, M. L., Meirink, J. A., van Veen, K., \& van Driel, J. H. (2017). Teachers' self-directed learning and teaching experience: What, how and why teachers want to learn. Teaching and Teacher Education. https://doi.org/10.1016/j.tate.2017.04.004.

Meister, D. G., \& Ahrens, P. (2011). Resisting plateauing: Four veteran teachers' stories. Teaching and Teacher Education: An International Journal of Research and Studies. https://doi.org/10.1016/j. tate.2011.01.002.

Morgado, J. C. (2017). Desafios curriculares para uma escola com futuro [Curricular challenges for a school with future]. ELO, 24, 37-44.

OECD (2010). Inspired by technology, driven by pedagogy. A systematic approach to technology-based school innovations. Educational Research and Innovation Series. [S.1.]: OECD.

OECD. (2019a). Education at a glance 2019: OECD indicators. Paris: OECD Publishing.

OECD. (2019b). Education at a glance 2019. Country note. Portugal. Pariss: OECD Publishing.

Orlando, J. (2014). Veteran teachers and technology: Change fatigue and knowledge insecurity influence practice. Teachers and Teaching. https://doi.org/10.1080/13540602.2014.881644.

Pereira, F. (2010). Infância, educação escolar e profissionalidade docente: Um mapeamento social dos discursos em formação inicial de professores. Lisboa: Fundação Calouste Gulbenkian.

Seaborn, K., \& Fels, D. I. (2015). Gamification in theory and action: A survey. International Journal of Human-Computer Studies. https://doi.org/10.1016/j.ijhcs.2014.09.006.

Schwab, K. (2016). Fourth industrial revolution. Genève: World Economic Forum.

Shifflet, R., \& Weilbacher, G. (2015). Teacher beliefs and their influence on technology use: A case study. Contemporary Issues in Technology and Teacher Education (CITE Journal), 15(3), 368-394.

Snoeyink, R., \& Ertmer, P. A. (2001). Thrust into technology: How veteran teachers respond. Journal of Educational Technology Systems. https://doi.org/10.2190/YDL7-XH09-RLJ6-MTP1.

Thomas, D., \& Brown, J. S. (2011). A new culture of learning. Cultivating the imagination for a world of constant change. [S. 1.]: CreateSpace.

Tuomi, I. (2006). The future of learning in the knowledge society: Disruptive changes for Europe by, 2020. In Y. Punie, et al. (Ed.), The Future of ICT and learning in the knowledge society (pp. 47-85). European Commission: Seville.

Yang, J., Pan, H., Zhou, W., \& Huang, R. (2018). Evaluation of smart classroom from the perspective of infusing technology into pedagogy. Smart Learning Environments. https://doi.org/10.1186/s4056 1-018-0070-1.

Zhu, Z.-T., Yu, M., \& Riezebos, P. (2016). A research framework of smart education. Smart Learning Environments. https://doi.org/10.1186/s40561-016-0026-2. 
Publisher's Note Springer Nature remains neutral with regard to jurisdictional claims in published maps and institutional affiliations. 